\title{
小麦籽粒淀粉分支酶同工酶结构组成及时空表达
}

\author{
刘正帅 ${ }^{1}$ 刘贵芬 ${ }^{1}$ 杨明煜 $^{2}$ 贾 晓 ${ }^{1}$ 李运祥 ${ }^{1}$ 赵法茂 ${ }^{1, *}$
}

${ }^{1}$ 泰山学院生物与酿酒工程学院, 山东泰安 $271021 ;{ }^{2}$ 泰安市第一中学, 山东泰安 271000

摘 要: 为阐明小麦支链淀粉合成的酶学机制, 以 8 个小麦品种的籽粒为材料, 采用非变性聚丙烯酰胺凝胶电泳 (Native-PAGE)和 SDS 聚丙烯酰胺凝胶电泳(SDS-PAGE)鉴定 SBE 同工酶类型、时空表达谱及亚基组成，分析 SBE 同 工酶空间分布特点和器官表达特异性。共检测到 4 种 SBE 同工酶, 其中 B 和 SBEIIa 分布在胚乳和叶片中, 而 A 和 $\mathrm{D}_{\mathrm{i}}$ 专一定位于胚乳中。在小麦籽粒灌浆过程中, $\mathrm{D}_{\mathrm{i}}$ 和 SBEIIa 首先表达, 而后是 $\mathrm{B}, \mathrm{A}$ 最后表达; 至灌浆末期, $\mathrm{B}$ 和 SBEIIa 停止表达。SBE 同工酶都是单亚基酶, 均由一条 86 92 kD 的多肽链组成。SBE 同工酶的空间分布具有器官 特异性，并在䊏粒发育进程中顺序表达。 $\mathrm{D}_{\mathrm{i}} 、 \mathrm{~B}$ 和 SBEIIa 是占主导地位的 SBE 同工酶，可能是决定 SBE 总酶活性的 主效应酶，在籽粒和叶片支链淀粉合成中起关键作用。

关键词: 小麦; 淀粉分支酶; 支链淀粉; 时空表达; 器官分布

\section{Constitution and Spatiotemporal Expression of Starch Branching Enzyme in Developing Wheat Grain}

\author{
LIU Zheng-Shuai ${ }^{1}$, LIU Gui-Fen ${ }^{1}$, YANG Ming-Yu ${ }^{2}$, JIA Xiao ${ }^{1}$, LI Yun-Xiang ${ }^{1}$, and ZHAO Fa-Mao ${ }^{1, *}$ \\ ${ }^{1}$ College of Biology and Enology, Taishan University, Tai'an 271021, China; ${ }^{2}$ Tai'an No.1 Middle School, Tai'an 271000, China
}

\begin{abstract}
This study aimed at disclosing the enzymatic mechanism in amylopectin synthesis in wheat (Triticum aestivum L.). The isozyme forms, organ localization, spatiotemporal expression profile and subunits constitution of starch branching enzyme (SBE) were identified in eight wheat cultivars from different provenances using native polyacrylamide gel electrophoresis (Native-PAGE) and SDS-PAGE. Four SBE isozymes were detected in wheat endosperm, in which isozymes B and SBEIIa were localized in endosperm and leaf, whereas isozymes $A$ and $D_{i}$ were exclusively present in endosperm. In the process of grain filling, $D_{i}$ and SBEIIa expressed first, followed by isozyme B, and isozyme A expressed finally. However, B and SBEIIa terminated to express at late filling stage. All SBE isozymes were composed of one subunit of $86-92 \mathrm{kD}$, and their spatial localization exhibited organ specificity. According to the expression level, $\mathrm{D}_{\mathrm{i}}, \mathrm{B}$, and SBEIIa are considered as dominant isozymes for grain endosperm development. They probably determinate the total SBE activity and serve as key factors in amylpectin biosynthesis in wheat grain and leaf.
\end{abstract}

Keywords: Triticum aestivum L.; Starch branching enzyme; Amylpectin; Spatiotemporal expression; Organ localization

淀粉是成熟小麦(Triticum aestivum L.)籽粒的主 要组成成分, 约占籽粒干重的 $70 \%$, 由直链淀粉和 支链淀粉两种糖元多聚体组成 ${ }^{[1]}$ 。研究证明, 直链淀 粉与支链淀粉的比例以及支链淀粉的精细结构显著 影响淀粉的理化性质, 进而决定面类制品, 尤其是 面条、馒头等东方食品的外观品质和食用品质 ${ }^{[2-3]}$ 。

直链淀粉是由 $\alpha-1,4$ 糖苷键连接成的线性分子,
很少或没有 $\alpha-1,6$ 糖苷键分支，由颗粒结合淀粉合酶 (GBSS，EC2.4.1.21) 催化合成; 支链淀粉则是由 $\alpha-1,4$ 糖苷键相连的葡萄糖链再通过 $\alpha-1,6$ 糖苷键连 接分支而成的葡萄糖聚合体，而淀粉分支酶(SBE， $\mathrm{EC} 2.4 .1 .18)$ 是唯一引入 $\alpha-1,6$ 糖苷键的淀粉合酶, 因 此其性质和活性是影响支链淀粉精细结构和含量的 重要因素 ${ }^{[4-7]}$ 。根据分子结构、免疫反应特性和底物

\footnotetext{
本研究由山东省自然科学基金项目(ZR2010CM034)和 2013 年全国大学生创新创业训练计划资助。

*通讯作者(Corresponding author): 赵法茂, E-mail: fmzhao8828@163.com

第一作者联系方式: E-mail: ZSLiu0508@163.com

Received(收稿日期): 2014-04-17; Accepted(接受日期): 2014-09-16; Published online(网络出版日期): 2014-09-26.

URL: http://www.cnki.net/kcms/detail/11.1809.S.20140926.0826.007.html
} 
特异性等, 将 SBE 分为 SBEI 和 SBEII 两种同工型。 玉米(Zea mays L.)体外实验表明, 与 SBEII 相比, SBEI 倾向于转移长的分支链, 当以直链淀粉为底物 时活性较高; 而 SBEII 倾向于转移短的分支链, 以 支链淀粉为底物时 SBEII 的活性高于 SBEI ${ }^{[8-9]}$ 。在 小麦籽粒发育过程中, 二者的表达时期也有差别, SBEII 从开花后第 13 天开始一直到第 34 天, 始终以 恒定水平表达, 而 SBEI 则在灌浆中期开始表达, 随 着灌浆进程的继续, 表达逐渐增强。小麦籽粒胚乳 中, SBEI 存在 4 种同工型, 即 $\mathrm{A} 、 \mathrm{~B} 、 \mathrm{D}_{\mathrm{i}}$ 和 $\mathrm{D}_{\mathrm{ii}}{ }^{[10]}$ 。 免疫杂交证明, SBEII 也有两种同工型, 即 SBEIIa 和 SBEIIb; SBEIIa 存在于胚乳的可溶性基质, 而 SBEIIb 结合在淀粉粒上 ${ }^{[11]}$ 。

Seo 等 ${ }^{[12]}$ 在葡聚糖分支酶缺失型酵母中表达玉 米的 SBE 同工型组合, 结果 SBEI 自身不能单独在 糖元合成中发挥作用, 只有在 SBEIIa和 SBEIIb 存在 的前提下才能行使功能; 而且 SBEII 在 SBEI 之前率 先作用于淀粉合成的前体引物, 可能 SBEII 同工型 作用的结果为 SBEI 提供底物。迄今为止在小麦中还 没有鉴定出自然发生的 SBEI 突变体, 只有 SBEII 突 变体才显示明显的淀粉表型效应。在水稻(Oryza sativa L.)中, ae (SBEIIb) 突变体可产生高直链淀粉 表型 ${ }^{[13]}$, 而在小麦中产生同样的表型效应需要同时 抑制 SBEIIa 和 SBEIIb 的表达 ${ }^{[14]}$ 。Schwall 等 ${ }^{[12]}$ 应用 反义 RNA 技术同时抑制马铃薯的 SBEII 和 SBEI 的 活性, 导致高直链淀粉含量表型, 而单独抑制 SBEI 却不能引起淀粉表型的显著改变。

目前, 究竟有多少种淀粉分支酶同工酶参与小
麦支链淀粉生物合成过程尚不明确，对 SBEI 和 SBEII 在支链淀粉合成中的作用机制不清楚, 而且 关于小麦 SBE 不同同工酶的时空表达谱和器官分 布特异性及酶亚基组成等也缺乏深入研究。本研究 选用 8 个小麦品种, 利用非变性聚丙烯酰胺凝胶电泳 (Native-PAGE)和 SDS 聚丙烯酰胺凝胶电泳 (SDSPAGE)，检测了 SBE 同工酶类型，鉴定了各同工酶 时空表达谱及器官表达特异性, 分析了其亚基组成 及结构, 以期阐明小麦不同器官支链淀粉合成的酶 偏好特点, 明确 SBE 同工酶结构组成, 旨在为小麦 品质改良提供理论依据。

\section{1 材料与方法}

\section{1 试验材料与取样方法}

不同种源地的 8 个小麦品种(表 1)均由山东省小 麦研究中心种质资源室保存并提供。2013 年 10 月 上旬, 将所有品种播种于山东农业大学教学实习农 场, 试验田块肥力均匀, 小区面积 $6 \mathrm{~m}^{2}, 3$ 次重复, 随机区组排列, 种植密度为 120 株 $\mathrm{m}^{-2}$, 其他田间常 规管理按小麦高产栽培技术规程进行。

于翌年小麦开花期选取生长均匀一致及穗大小 相近的植株，挂牌标记同一日开花的麦穗，于开花 后第 5 天开始取样, 之后每隔 $5 \mathrm{~d}$ 取样一次, 直至开 花后第 35 天完全成熟。取样时间为 9:00-11:00, 每 品种取 3 5 个标记穗及同株旗叶, 每穗取中部小穗 籽粒, 籽粒和旗叶经液氮速冻 $30 \mathrm{~min}$ 后放入 $-80^{\circ} \mathrm{C}$ 超低温冰箱中保存，用于聚丙烯酰胺凝胶电泳和酶 活性测定。

表 1 供试小麦品种成熟籽粒淀粉含量

Table 1 Starch contents of wheat cultivars tested at maturity stage

\begin{tabular}{|c|c|c|c|c|c|}
\hline $\begin{array}{l}\text { 组别 } \\
\text { Group }\end{array}$ & $\begin{array}{c}\text { 品种 } \\
\text { Cultivar }\end{array}$ & $\begin{array}{c}\text { 支链淀粉含量 } \\
\text { Amylopectin content (\%) }\end{array}$ & $\begin{array}{c}\text { 直链淀粉含量 } \\
\text { Amylose content (\%) }\end{array}$ & $\begin{array}{c}\text { 总淀粉含量 } \\
\text { Starch content }(\%)\end{array}$ & $\begin{array}{c}\text { 支/直比 } \\
\text { Amylopectin/amylose ratio }\end{array}$ \\
\hline \multirow{4}{*}{$\begin{array}{c}\text { 低支链淀粉 } \\
\text { Low amylopectin }\end{array}$} & 鲁麦 18 Lumai 21 & 52.85 & 25.45 & 78.30 & 2.08 \\
\hline & $\begin{array}{l}\text { 山东辐 } 63 \\
\text { Shandongfu } 63\end{array}$ & 52.41 & 20.15 & 72.56 & 2.60 \\
\hline & 济南 17 Jinan 17 & 53.34 & 19.08 & 72.42 & 2.80 \\
\hline & 周麦 19 Zhoumai 19 & 54.87 & 22.33 & 77.20 & 2.46 \\
\hline \multirow{4}{*}{$\begin{array}{c}\text { 高支链淀粉 } \\
\text { High amylopectin }\end{array}$} & 鄂麦 6 号 Emai 6 & 62.48 & 17.18 & 79.66 & 3.64 \\
\hline & $\begin{array}{l}\text { 陕旱 } 8675 \\
\text { Shaanhan } 8675\end{array}$ & 61.12 & 20.75 & 81.87 & 2.95 \\
\hline & 济南 16 Jinan 16 & 61.22 & 17.67 & 78.89 & 3.46 \\
\hline & 石特 14 Shite 14 & 60.81 & 18.69 & 79.50 & 3.25 \\
\hline
\end{tabular}




\section{2 测定项目与方法}

\subsection{1 粗酶液提取}

参照 Yamanouchi 和 Nakamura ${ }^{[15]}$ 的方法, 稍作修改。称取 $0.4 \mathrm{~g}$ 左右的 小麦籽粒, 加 $1 \mathrm{~mL}$ 提取液(含 $50 \mathrm{mmol} \mathrm{L}^{-1}$ HEPES$\mathrm{NaOH}, \mathrm{pH}$ 7.5, $5 \mathrm{mmol} \mathrm{L}^{-1}$ EDTA, $1 \mathrm{mmol} \mathrm{L}^{-1}$ DTT, 2 $\mathrm{mmol} \mathrm{L}^{-1} \mathrm{KCl}, 1 \%$ 聚乙烯吡咯烷酮 K-30)研磨成匀浆, 倒入 $5 \mathrm{~mL}$ 离心管内, 再用 $3 \mathrm{~mL}$ 提取液分 2 次冲洗 研钵, 冲洗液一并倒入离心管, $4^{\circ} \mathrm{C}$ 下 $15000 \times g$ 离心 $15 \mathrm{~min}$, 上清液即为粗酶液。

1.2.2 SBE 活性测定 参照李太贵等 ${ }^{[16]}$ 的方法, 并作适当改进。将粗酶液稀释 10 倍, 取 $100 \mu \mathrm{L}$ 稀释 液, 加 $1280 \mu \mathrm{L}$ 提取缓冲液及 $0.75 \%$ 可溶性淀粉 120 $\mu \mathrm{L}$, 混匀, $37^{\circ} \mathrm{C}$ 恒温水浴保温 $20 \mathrm{~min}$ 后置沸水浴 1 $\mathrm{min}$ 终止反应, 加 $2 \mathrm{~mL}$ 水(含 $0.2 \%$ 浓 $\mathrm{HCl}$ ) 稀释, 再 加 $150 \mu \mathrm{L}$ 碘液显色 $10 \mathrm{~min}$ 后在 $660 \mathrm{~nm}$ 波长下测 $\mathrm{OD}$ 值。另取一只试管, 加入粗酶液后立即置沸水浴 1 $\mathrm{min}$ 使酶灭活, 再按以上步骤加入各种溶液, 作为对 照。分支酶活性以 $660 \mathrm{~nm}$ 波长的吸光度下降百分率 表示, 以每降低 $1 \%$ 碘蓝值为一个酶活性单位。所有 测定均重复 3 次。

\subsubsection{Native-PAGE 和酶活性检测 粗酶液参照} Nagamine 等 ${ }^{[17]}$ 的方法, 参照 Davis ${ }^{[18]}$ 的非变性聚丙 烯酰胺凝胶电泳方法。分离胶浓度 $8.5 \%$, 浓缩胶浓 度 $5 \%$, 恒流 $20 \mathrm{~mA}$, 所有操作均在 $0 \sim 4{ }^{\circ} \mathrm{C}$ 下进行。 参照 Nagamine 等 ${ }^{[17]}$ 的方法进行酶活性染色。SBE 非变性聚丙烯酰胺凝胶电泳及酶活性染色, 是基于 淀粉遇磑显色原理。SBE 催化分支的支链淀粉遇碘 呈现棕褐色, 而磷酸化酶催化合成的线性直链淀粉 则被染成蓝色。染色后按 Morell 等 ${ }^{[10]}$ 的方法对 SBE 同工酶谱带进行位点判读和命名, 酶带浓度即代表 酶活性大小。另外, 应用 BandScan 5.0 凝胶分析软 件对电泳图谱进行定量扫描。

\section{3 目标蛋白回收及其 SDS-PAGE}

参照 Hager 等 ${ }^{[19]}$ 和 Dynan 等 ${ }^{[20]}$ 的方法回收目标 蛋白。凝胶板活性染色后, 用刀片切下 $2 \mathrm{~mm} \times 8 \mathrm{~mm}$ 目的条带放入干净试管, 加 $1 \mathrm{~mL}$ 双蒸水(含 $1 \mathrm{mmol}$ $\mathrm{L}^{-1} \mathrm{DTT}$ ), 静置 $15 \mathrm{~min}$, 其间更换一次缓冲液; 倒掉 缓冲液, 加 $1 \mathrm{~mL}$ 洗提 buffer $\left[50 \mathrm{mmol} \mathrm{L}{ }^{-1}\right.$ Tris-HCl, pH 7.5, 0.1\% SDS, $1 \mathrm{mmol} \mathrm{L}^{-1}$ EDTA, $5 \mathrm{mmol} \mathrm{L}^{-1}$ DTT, $150 \mathrm{mmol} \mathrm{L}^{-1} \mathrm{NaCl}, 0.1 \mathrm{mg} \mathrm{mL}^{-1}$ 牛血清白蛋白 (BSA)], 捣碎凝胶并在 $25^{\circ} \mathrm{C}$ 过夜洗提; 于 $4^{\circ} \mathrm{C}$ 下 $15000 \times g$ 离心 $5 \mathrm{~min}$ 以沉淀破碎凝胶, 将上清液转 入另一个干净试管。

上清液加 4 倍体积冷丙酮 $\left(-20^{\circ} \mathrm{C}\right)$, 于 $-20^{\circ} \mathrm{C}$ 放置
$30 \mathrm{~min}, 4^{\circ} \mathrm{C}$ 下 $15000 \times g$ 离心 $10 \mathrm{~min}$, 去丙酮上清液, 于室温下干燥沉淀, 并在 $100 \mu \mathrm{L}$ 样品 buffer (62.5 $\mathrm{mmol} \mathrm{L}{ }^{-1}$ Tris- $\mathrm{HCl}, \mathrm{pH} 6.8,2 \% \mathrm{SDS}, 5 \%$ 颈基乙醇, $10 \%$ 甘油)中重悬, 最后上样电泳。

参照 Laemmli ${ }^{[21]}$ 的方法进行 SDS-PAGE，分离 胶浓度 $10 \%$, 浓缩胶浓度 5\%, 恒流 $25 \mathrm{~mA}$ ，上样量 $20 \mu \mathrm{L}$ ，银染色。

\section{2 结果与分析}

\section{1 小麦灌浆过程中 SBE 活性变化}

选择 2 个低支链淀粉和 1 个高支链淀粉品种测 定灌浆过程中 SBE 活性动态变化, 发现 SBE 活性呈 单峰曲线变化(图 1)。酶活性在灌浆初期较低, 随灌 浆进程逐渐提高, 至开花后 $20 \mathrm{~d}$ 达到峰值, 之后逐 渐下降，在灌浆后期还保持一定活性。3 个品种之间 变化趋势基本一致, 且酶活性大小差异不显著。

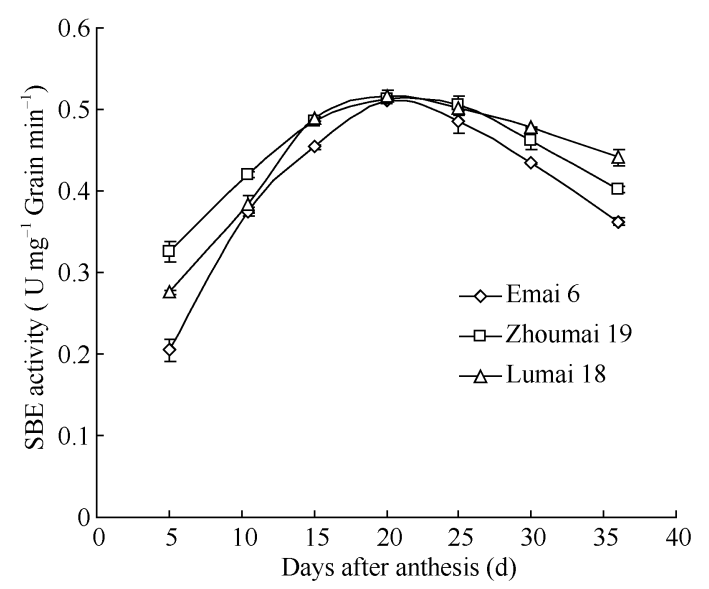

图 1 不同品种小麦花后籽粒中淀粉分支酶活性的动态变化 Fig. 1 Changes of SBE activity in different wheat cultivars during grain filling

2.2 不同灌浆阶段籽粒中 SBE 同工酶及其活性 花后 $5 \mathrm{~d}$, 从 8 个供试品种中共鉴定出 2 种同工 酶, 分别命名为 $D_{i}$ 和 SBEIIa ${ }^{[10]}$ 。电泳图谱显示, SBEIIa 迁移率最快, 表明其携带的负电荷最多; $D_{i}$ 迁移率较慢, 表明所携带负电荷较少, 根据谱带浓 度, $D_{i}$ 表达量较 SBEIIa 略高(图 2-a)。由于灌浆初期, 二者的活性较低, 淀粉的分支化程度亦低, 因此其 谱带均被染成蓝色。另外, 8 个品种均表达相同的同 工酶类型, $\mathrm{D}_{\mathrm{i}}$ 和 SBEIIa的迁移率在品种间高度一致。 因此，在灌浆初期，小麦籽粒中表达了至少 2 种 SBE 同工酶，以启动灌浆早期的淀粉合成。

花后 $20 \mathrm{~d}, \mathrm{D}_{\mathrm{i}}$ 和 SBEIIa 继续稳定表达，同时，同 工酶 $\mathrm{A}$ 和 $\mathrm{B}$ 亦表达(图 2-b)。从酶带浓度上可以判断, 
同工酶 $\mathrm{D}_{\mathrm{i}}$ 表达量较大, 酶活性最高, $\mathrm{B}$ 和 SBEIIa 位 居其次, 且几乎所有品种均具有这 3 种同工酶, 暗 示着其可能是小麦籽粒中对 SBE 活性贡献率最大的 同工酶形式。值得注意的是, 鲁麦 18 缺失同工酶 B, 但表达了一条新的、携带负电荷较少的谱带, 与其 他 7 个品种的同工酶类型稍有不同。

花后 $30 \mathrm{~d}$ ，表达的 SBE 同工酶种类与花后 $20 \mathrm{~d}$ 完全相同, 没有发现同工酶种类减少(图 2-c)。与花后 $20 \mathrm{~d}$ 稍有不同的是, 酶带浓度变淡, 强度变细。说明 在小麦灌浆后期，随着淀粉合成速率减慢，虽然各同 工酶类型和数量不减少, 但其酶活性已显著降低。

2.3 小麦灌浆过程中籽粒和叶片 SBE 同工酶活 性动态分析

\subsection{1 籽粒 SBE 同工酶活性动态 以陕旱 8675}

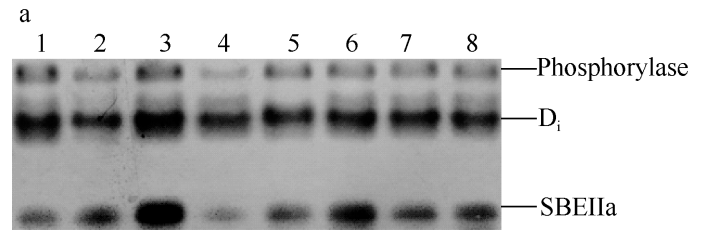

为例, SBE 同工酶谱带的浓度随灌浆进程而变化(图 3-a), 且与 SBE 酶活性动态变化(图 1)升降走势相契 合。花后 $5 \mathrm{~d}$ 和 $10 \mathrm{~d}$ 时各同工酶谱带颜色较淡, 表 达量较小; 花后 $15 \mathrm{~d}$ 左右时染色明显变浓, 花后 $20 \mathrm{~d}$ 时表达量接近峰值, 20 25 d 的表达量差异不明 显; 从 $30 \mathrm{~d}$ 开始表达量明显降低; 至 $35 \mathrm{~d}$ 灌浆结束, 酶带颜色又显著变淡。

SBE 同工酶在灌浆过程中顺序表达。花后 $5 \mathrm{~d}$ 只有 $\mathrm{D}_{\mathrm{i}}$ 和 SBEIIa 表达，表达量较低; 花后 $10 \mathrm{~d}$ ，同 工酶 $\mathrm{B}$ 开始表达, 此时三者的酶活性较低, 表达量 都不高; 花后 $15 \mathrm{~d}$ ，同工酶 $\mathrm{A}$ 开始表达，但表达量较 低，而其他各同工酶表达量明显增加，这种表达谱 一直持续到花后 $25 \mathrm{~d}$; 花后 $30 \mathrm{~d}$ ，各同工酶表达量 稍微下降，但同工酶种类不减少; 至花后 $35 \mathrm{~d}$ 灌浆 b

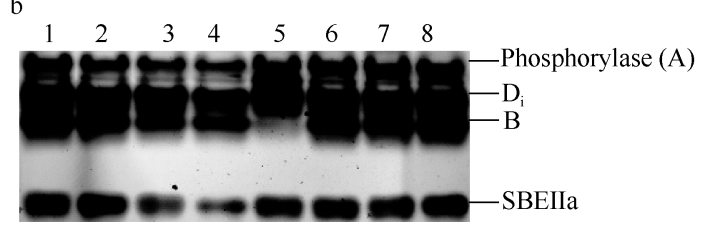

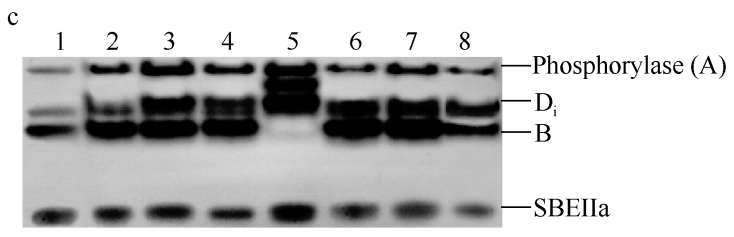

图 2 小麦不同灌浆阶段䊏粒 SBE 同工酶的 Native-PAGE 图谱

Fig. 2 Native-PAGE profile of SBE isozymes in wheat grain during filling period

$\mathrm{a}$ : 开花后 $5 \mathrm{~d}$; b: 开花后 $20 \mathrm{~d}$; : 开花后 $30 \mathrm{~d}$ 。花后 $5 \mathrm{~d}$, 同工酶 $\mathrm{A}$ 未表达(酶活性染色为蓝色), 第一条带为内源磷酸化酶; 花后 $20 \mathrm{~d}$ 和 $30 \mathrm{~d}$, 同工酶 $\mathrm{A}$ 已经表达(酶活性染色为褐色), 但与内源磷酸化酶带重叠。1: 鄂麦 6 号; 2: 陕旱 8675; 3: 济南 16; 4: 石特 14; 5: 鲁 麦 18; 6: 山东辐 63; 7: 济南 17; 8: 周麦 19。

a: at $5 \mathrm{~d}$ after anthesis (DAA); b: at 20 DAA; c: at 30 DAA. At 5 DAA, the most upper band shows phosphorylase because isozyme A was not expressed (The color of band was blue). At 20 DAA and 30 DAA, the most upper band shows the superposed phosphorylase and SBE isozyme A (Enzyme activity staining showed brown). 1: Emai 6; 2: Shaanhan 8675; 3: Jinan 16; 4: Shite 14; 5: Lumai 18; 6: Shandongfu 63; 7: Jinan 17; 8: Zhoumai 19.
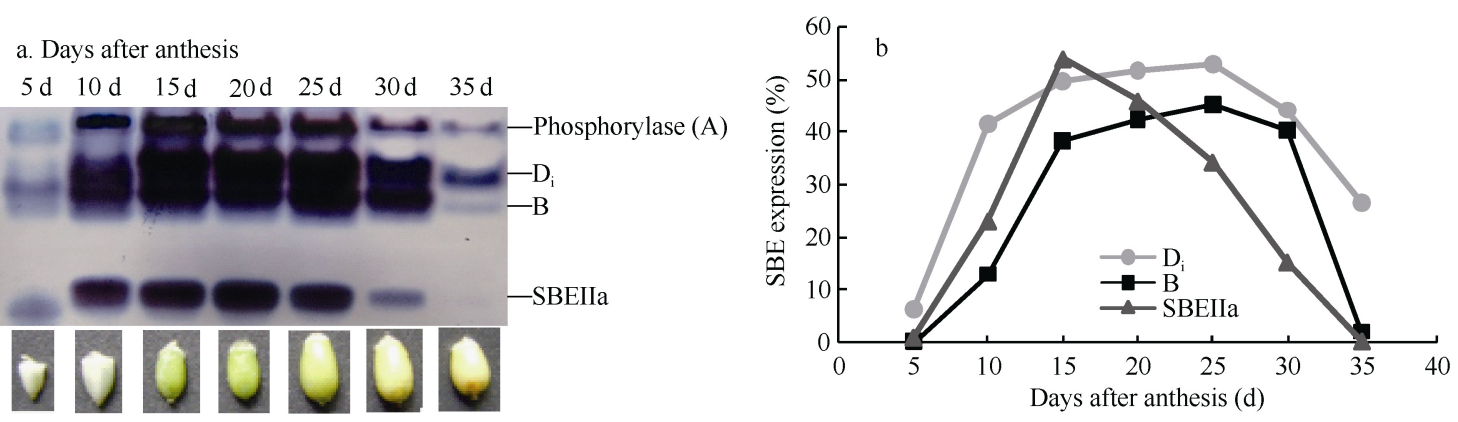

图 3 Native-PAGE 检测陕旱 8675 灌浆期籽粒 SBE 同工酶表达及活性

Fig. 3 Native-PAGE profile of SBE isozymes and SBE expression quantity in Shaanhan 8675 during grain filling a: 花后 $5 \mathrm{~d}$ 和 $10 \mathrm{~d}$, 同工酶 $\mathrm{A}$ 尚未表达, 谱带为内源磷酸化酶(酶带染色为蓝色); 花后 $15 \mathrm{~d}, \mathrm{~A}$ 开始表达, 谱带为 $\mathrm{A}$ 和内源磷酸化酶的 叠加(褐色和蓝色叠加)。 b: SBE 同工酶表达量用灰度值 $(\%)$ 表示; 同工酶 A 因无法与内源磷酸化酶分离而未测定。 a: The bands of phosphorylase and isozyme A were superposed in Native-PAGE. Isozyme A was not expressed in the developing grain at 5 and 10 days after anthesis because enzyme activity staining showed blue. but expressed since 15 DAA (Overlapping blue and brown). b: Expression quantities of SBE isozymes were represented by gray value (\%). Data of isozyme A were not available due to unable to separate it from phosphorylase. 
结束, 各同工酶表达量明显降低, $\mathrm{D}_{\mathrm{i}}$ 继续存在, $\mathrm{B}$ 表 达信号非常微弱, SBEIIa 已经检测不到, 即已停止 表达(图 3-a,b)。

2.3.2 叶片 SBE 同工酶活性动态花后 $10 \mathrm{~d}$, 旗 叶中仅表达 B 和 SBEIIa 两种 SBE 同工酶, 且 SBEIIa 表达量较高, 活性较强。值得注意的是, 鲁麦 18 缺 之 $\mathrm{B}$ 同工酶, 但表达了一条活性很弱的 $\mathrm{D}_{\mathrm{i}}$ 同工酶, 这与籽粒灌浆高峰期的 SBE 表达谱相类似, 但又不

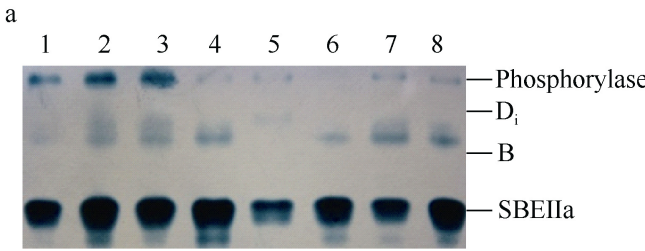

完全相同(图 4-a)。

将花后 $10 \mathrm{~d}$ 的小麦籽粒与其叶片的 SBE 放在一 起电泳比对, 二者的 SBE 无论是表达量还是表达类 型都具有显著差异。从表达量看, 籽粒中的 SBE 各 同工酶条带浓而粗，而叶片中的谱带淡而细，说明 籽粒酶活性远大于叶片; 从表达类型上看, 花后 $10 \mathrm{~d}$ 的籽粒已表达 $\mathrm{D}_{\mathrm{i}} 、 \mathrm{~B}$ 和 SBEIIa 三种同工酶，但 叶片中仅同工酶 B 有弱表达(图 4-b)。

图 4 小麦旗叶中 SBE 同工酶的 Native-PAGE 谱

Fig. 4 Native-PAGE profile of SBE isozymes in wheat flag leaf

$\mathrm{a}$ : 花后 $10 \mathrm{~d}$ 旗叶; $\mathrm{b}$ : 花后 $10 \mathrm{~d}$ 旗叶与籽粒对比。1: 鄂麦 6 号; 2 : 陕旱 8675; 3: 济南 $16 ; 4$ : 石特 $14 ; 5$ : 鲁麦 18; 6: 山东辐 63; 7: 济南 $17 ; 8$ : 周麦 19 。S: 籽粒; $\mathrm{L}$ : 叶片。

a: Flag leaf sampled at $10 \mathrm{~d}$ after anthesis (DAA); b: Comparison of expressions between leaf and seed at 10 DAA. 1: Emai 6;

2: Shaanhan 8675; 3: Jinan 16; 4: Shite 14; 5: Lumai 18; 6: Shandongfu 63; 7: Jinan 17; 8: Zhoumai 19. S: seed; L: leaf.

2.4 小麦胚乳 SBE 各同工酶亚基组成鉴定

SBE 活性染色后, 把电泳条带切下, 将蛋白质 回收并进行 SDS-PAGE。结果显示, $\mathrm{D}_{\mathrm{i}} 、 \mathrm{~B}$ 和 SBEIIa 同工酶变性后均显示一条谱带, 分子量相近, 大约 86 88 kD (图 5), 这与前人的研究结果 ${ }^{[10]}$ 基本一致。 另外, 在内源磷酸化酶条带位置, 回收了一条比上 述 3 种同工酶稍为细弱的蛋白带, 为内源磷酸化酶 或同工酶 $\mathrm{A}$, 分子量大约为 $92 \mathrm{kD}$ (图 5)。

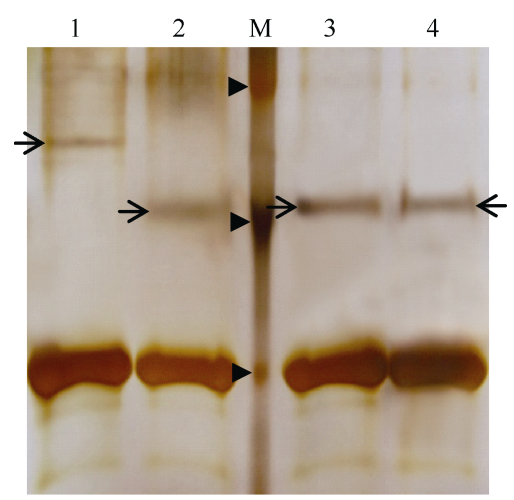

图 5 小麦胚乳中 SBE 同工酶 SDS-PAGE 分析

Fig. 5 SDS-PAGE profile of SBE isozymes in wheat endosperm

$\mathrm{M}$ : 蛋白质分子量标准, 3 条带从上到下分别指示 $120 \mathrm{kD} 、 85 \mathrm{kD}$ 和牛血清白蛋白(BSA); 1 : 内源磷酸化酶或同工酶 $\mathrm{A} ; 2: \mathrm{D}_{\mathrm{i}}$;

3: 同工酶 B; 4: SBEIIa。箭头指示回收蛋白质谱带。

M: Protein standard ladder, showing the positions of $120 \mathrm{kD}, 85 \mathrm{kD}$, and BSA (from top to bottom); 1: Endogenous phosphorylase or isozyme $A ; 2: D_{i} ; 3$ : isozyme $B ; 4$ : SBEIIa. The arrows show band patterns of the retrieved proteins.

\section{3 讨论}

Hazard 等 ${ }^{[22]}$ 利用 RNA 干涉技术在四倍体小麦 中产生 SBEIIa-A 和 SBEIIa-B 双突变, 结果发现, 突 变体的直链淀粉含量增加 $22 \%$ 。在大麦中, Carciofi 等 ${ }^{[23]}$ 通过协同沉默所有 SBE 基因也得到相同研究结 果, 导致籽粒胚乳内只产生直链淀粉。本试验表明, 在小麦灌浆的高峰期, $D_{i} 、 B$ 和 SBEIIa 大量表达, 酶 活性最大。可以判断, 同工酶 $\mathrm{D}_{\mathrm{i}} 、 \mathrm{~B}$ 和 SBEIIa, 尤其 $\mathrm{D}_{\mathrm{i}}$ 和 SBEIIa 是籽粒胚乳中占主导地位的 SBE 同工酶 形式, 很可能是决定 SBE 总酶活性的主效应酶, 在灌 浆高峰期和灌浆后期籽粒淀粉合成中起关键作用。

小麦籽粒胚乳中存在不同形式的 SBE 同工酶, 但在整个灌浆进程中，其表达顺序不同，暗示灌浆 不同时期籽粒内淀粉的合成是由不同同工酶所催化 完成的。因此小麦籽粒发育的不同时期，可通过选 择不同的同工酶系统启动淀粉合成过程。鲁麦 18 在 灌浆高峰期和叶片中均缺失 B 同工酶, 这可能是不 同品种之间的差异和遗传多态性表现。另外，鲁麦 18 的支链淀粉含量较低, 而直链淀粉含量最高, 这 是否是由于缺失 $\mathrm{B}$ 同工酶导致的结果, 目前这种相 关性尚不能确定。

一般认为, 小麦叶片负责临时性淀粉的合成, 而籽粒胚乳㫟藏淀粉合成的原料来自于叶片中的淀 粉或其降解产物——蔗糖。本研究发现, SBE 同工酶 
在叶片和籽粒中的空间分布具有器官特异性, 存在 于籽粒中的 $\mathrm{D}_{\mathrm{i}}$ 和 $\mathrm{A}$ 同工酶在叶片中却检测不到其表 达, 说明催化临时淀粉和咜藏淀粉的合成具有不同 的酶偏好。暗示叶片和胚乳中参与支链淀粉合成的 主效应酶类是不同的, 可能临时性淀粉和永久性淀 粉的合成机制本身就存在差别, 叶和籽粒通过选择 不同的 SBE 酶系统，启动各自支链淀粉的合成。

按照 Morell 等 ${ }^{[10]}$ 对 SBE 各同工酶的位点判读方 法, SBEI 应该有 4 种同工酶, 即 $\mathrm{A} 、 \mathrm{D}_{\mathrm{i}} 、 \mathrm{D}_{\mathrm{ii}}$ 和 $\mathrm{B}$ 。 但是, 本实验在所有 8 个供试品种中, 没有检测到 $\mathrm{D}_{\mathrm{ii}}$ 同工酶的表达。这可能是不同品种之间遗传多态 性所致。同时也说明, 并不是所有小麦品种都具有 $\mathrm{D}_{\mathrm{ii}}$ 同工酶。可能的情况是, $\mathrm{D}_{\mathrm{ii}}$ 并不是 $\mathrm{SBE}$ 的主要形 式，在支链淀粉合成中只起辅助作用。

Morell 等 ${ }^{[10]}$ 和 Rahman 等 ${ }^{[24]}$ 研究发现, 小麦开 花后 13 34 d, SBEII 始终以恒定水平表达, 是淀粉 分支酶中最基本的组成性同工型, 而 SBEI 直到花后 $18 \mathrm{~d}$ 才检测到其表达, 可能在籽粒发育后期淀粉合 成中发挥重要作用。本研究结果与此稍有不同, 无 论是 SBEI 还是 SBEII, 花后 $5 \mathrm{~d}$ 已开始表达, 花后 $10 \mathrm{~d}$ 已全部表达。另外, SBEII 在灌浆末期表达量降 低直至最终消失。其可能的原因是对 SBE 同工酶表 达的检测时间不同, Morell 等 ${ }^{[10]}$ 和 Rahman 等 ${ }^{[24]}$ 是从 花后第 13 天开始取样, 而本试验则从花后第 5 天开 始。此外, 试验材料、场所和当地栽培条件的差异 及籽粒发育进程的不同也可能导致结果误差。

研究证明, 在谷物体内参与淀粉合成的酶可能 以蛋白质复合体的形式行使功能，其中一个酶的缺 失将会导致复合体其他成员的活性钝化而降低或失 去活性。另外许多淀粉合酶突变体的研究表明, 单 个基因的突变往往导致其他淀粉合酶活性变化的多 重效应。例如, 小麦磷酸化酶、SBEI 和 SBEIIb 可形 成多蛋白复合体 ${ }^{[25-27]}$ 。但本研究并未检测到这种多 酶复合体。首先这种多酶复合体可能只存在于植物 体内, 一旦脱离体内环境, 复合体即解聚合; 其次, 复合体中蛋白质之间相互结合的化学键都是一些非 共价键, 键合力弱, 即使提取液中有复合体存在, 在电场力作用下，已经被分离成单个独立酶。同时 也说明，在体外淀粉合成中, SBE 单个同工酶即可催 化淀粉合成。本试验结果支持上述分析, 同工酶 $\mathrm{D}_{\mathrm{i}}$ 、 $B$ 和 SBEIIa 在 SDS-PAGE 中均显示单一谱带, 且分 子量大体一致，说明上述 3 种同工酶都由一条多肽 链组成, 是单亚基酶。至于同工酶 $\mathrm{A}$, 由于其和内源
磷酸化酶的分子形状和大小及所带电荷极其接近, 以至于二者在 Native-PAGE 中的迁移率相同, 不易 分开。因此, 在凝胶板中 $\mathrm{A}$ 条带位置, 回收的一条 分子量大约为 $92 \mathrm{kD}$ 的蛋白带可能是同工酶 $\mathrm{A}$ 和内 源磷酸化酶的重叠带。

\section{4 结论}

小麦植株中共表达 4 种 SBE 同工酶, 其中 B 和 SBEIIa 分布在胚乳和叶片中, 而 $\mathrm{A}$ 和 $\mathrm{D}_{\mathrm{i}}$ 仅定位于胚 乳中。在籽粒灌浆过程中 SBE 同工酶表达具有顺序 性。 $\mathrm{SBE}$ 同工酶均为单亚基酶, 分子量约为 86 92 $k D 。 D_{i} 、 B$ 和 SBEIIa 是占主导地位的 SBE 同工酶，在 籽粒和叶片支链淀粉合成中起关键作用。

\section{References}

[1] Baga M, Glaze S, Mallard C S, Chibbar R. A starch branching enzyme gene in wheat produces alternatively spliced transcripts. Plant Mol Biol, 1999, 40: 1019-1030

[2] 姚大年, 李保云, 朱金宝, 梁荣奇, 刘广田. 小麦品种主要淀 粉性状及面条品质预测指标的研究. 中国农业科学, 1999, 32(6): 84-88

Yao D N, Li B Y, Zhu J B, Liang R Q, Liu G T. Study on main starch properties and predictive indexes of noodle quality in common wheat. Sci Agric Sin, 1999, 32(6): 84-88 (in Chinese with English abstract)

[3] McCormick K M, Panozzo J F, Hong S H. A swelling power test for selecting potential noodle quality wheats. Aust J Agric Res, 1991, 42: 317-323.

[4] Myers A M, Morell M K, James M G, Ball S G. Recent progress toward understanding the amylopectin crystal. Plant Physiol, 2000, 122: 989-997

[5] Kossmann J, Lloyd J. Understanding and influencing starch biochemistry. Crit Rev Plant Sci, 2000, 19: 171-226

[6] James M G, Denyer K, Myers A M. Starch synthesis in the cereal endosperm. Curr Opin Plant Biol, 2003, 6: 215-222

[7] Jeon J S, Ryoo N, Hahn T R, Walia H, Nakamura Y. Starch biosynthesis in cereal endosperm. Plant Physiol Biochem, 2010, 48: 383-392

[8] Guan H P, Preiss J. Differentiation of the properties of the branching isozymes from maize (Zea mays). Plant Physiol, 1993, 102: $269-1273$

[9] Takeda Y, Guan H P, Preiss J. Branching of amylose by the branching isoenzymes of maize endosperm. Carbohydr Res, 1993 , 240: 253-263

[10] Morell M K, Blennow A, Hashemi B K, Samuel M S. Differential expression and properties of starch branching enzyme isoforms in developing wheat endosperm. Plant Physiol, 1997, 113: 201-208

[11] Regina A, Kosar H B, Li Z, Pedler A, Mukai Y, Yamamoto M, Gale K, Sharp P J, Morell M K, Rahman S. Starch branching enzyme IIb in wheat is expressed at low levels in the endosperm compared to other cereals and encoded at a non-syntenic locus. Planta, 2005, 222: 899-909 
[12] Schwall G P, Safford R, Westcott R J, Jeffcoat R J, Tayal A, Shi Y C, Gidley M G, Jobling S A. Production of very-high-amylose potato starch by inhibition of SBE A and SBE B. Nation Biotechnol, 2000, 18: 551-554

[13] Nishi A, Nakamura Y, Tanaka N, Satoh H. Biochemical and genetic effects of amylose-extender mutation in rice endosperm. Plant Physiol, 2001, 127: 459-472

[14] Regina A, Bird D, Topping D, Bowden S, Freeman J, Barsby T, Hashemi B K, Li Z, Rahman S, Morell M. High amylose wheat generated by RNA-interference improves indices of large bowel health in rats. Proc Natl Acad Sci USA, 2006, 103: 3546-3551.

[15] Yamanouchi H, Nakamura Y. Organ specificity of isoforms of starch branching enzyme (Q-enzyme) in rice. Plant Cell Physiol, 1992, 33: 985-991

[16] 李太贵, 沈波, 陈能, 罗玉坤. Q 酶对水稻籽粒严白形成的影 响. 作物学报, 1997, 23: 338-344

Li T G, Shen B, Chen N, Luo Y K. Effect of Q-enzyme on the chalkiness formation of rice grain. Acta Agron Sin, 1997, 23: 338-344 (in Chinese with English abstract)

[17] Nagamine T, Yoshida H, Komae K. Varietal differences and chromosome locations of multiple isoforms of starch branching enzyme in wheat endosperm. Phytochemistry, 1997, 46: 23-26

[18] Davis B J. Disc electrophoresis II method and application of human serum proteins. Ann NY Acad Sci, 1964, 121: 404-427

[19] Hager D A, Burgess R R. Elution of proteins from SDSpolyacrylamide gels, removal of SDS, and renaturation of enzymatic activity: results with sigma subunit of $E$. coli RNA polymerase, wheat germ DNA topoisomerase, and other enzymes. Anal Biochem, 1980, 109: 76-86
[20] Dynan W S, Jendrisak J J, Hager D A, Burgess R D. Purification and characterization of wheat germ DNA topoisomerase I (nicking-closing enzyme). J Biol Chem, 1981, 256: 5860-5865

[21] Laemmli U K. Cleavage of structural proteins during the assembly of the head of bacteriophage T4. Nature, 1970, 227: 680-685

[22] Hzard B, Zhang X Q, Colasuonno P, Uauy C, Beckles D M, Dubcovsky J. Induced mutations in the starch branching enzyme II (SBEII) genes increase amylase and resistant starch content in durum wheat. Crop Sci, 2012, 52: 1754-1766

[23] Carciofi M, Blennow A, Jensen S L, Shaik S S, Henriksen A, Buleon A, Holm P B, Hebelstrup K. Concerted suppression of all starch branching enzyme genes in barley produces amylase-only starch granules. BMC Plant Biol, 2012, 12: 223-239

[24] Rahman S, Hashemi B K, Samuel M S, Hill A, Abbott D C, Skerritt J H, Preiss J, Appels R, Morell M K. The major proteins of wheat endosperm starch granules. Aust J Plant Physiol, 1995, 22: 793-803

[25] Tetlow I J, Wait R, Lu Z X, Akkasaeng R, Bowsher C G, Esposito S, Hashemi B K, Morell M K, Emes M J. Protein phosphorylation in amyloplasts regulates starch branching enzyme activity and protein-protein interactions. Plant Cell, 2004, 16: 694-708

[26] Tetlow I J, Beisel K G, Cameron S, Makhmoudova A, Liu F, Bresolin N S, Wait R, Morell M K, Emes M J. Analysis of protein complexes in wheat amyloplasts reveals functional interactions among starch biosynthetic enzymes. Plant Physiol, 2008, 146: 1878-1891

[27] Hennen-Bierwagen T A, Liu F, Marsh R S, Kim S, Gan Q, Tetlow I J, Emes M J, James M G, Myers A M. Starch biosynthetic enzymes from developing maize endosperm associate in multisubunit complexes. Plant Physiol, 2008, 146: 1892-1908 